\title{
Synthesis and characterization of nanocrystalline tungsten oxide nanosheets in large scale
}

\author{
Rong $\mathrm{Hu}$, Huasheng $\mathrm{Wu},{ }^{\text {a) }}$ and Kunquan Hong \\ Physics Department and the University of Hong Kong-Chinese Academy of Sciences (HKU-CAS) Joint \\ Laboratory on New Materials, The University of Hong Kong, Hong Kong, Peoples Republic of China
}

(Received 3 July 2008; accepted 23 September 2008)

\begin{abstract}
A high quantity of tungsten oxide nanosheets were synthesized by oxidizing tungsten plates with potassium hydrate as the catalyst and tungsten plate as the substrate. The structural and geometrical properties were characterized by various techniques. It was found that the crystalline nanosheets have a $\mathrm{WO}_{3}$ structure with thicknesses of $30-50 \mathrm{~nm}$ and widths up to tens of micrometers. There exist two characteristic acute angles of about $37^{\circ}$ or $51^{\circ}$ on the nanosheet plane. The formation of these angles and the growth mechanism were discussed.
\end{abstract}

\section{INTRODUCTION}

Tungsten oxide has attracted extensive attention over the last decade because of its interesting electrochromic, photochromic, and gaschromic properties. It has now become a promising candidate of the transition metal oxides that are widely used in industry applications, especially in the applications of field emissions, electrochromic materials, and gas sensors. ${ }^{1-4}$ Various tungsten oxide nanostructures have been synthesized, such as nanowires, nanotubes, nanonetworks, and nanoribbons. ${ }^{5-15}$ Among the synthesizing techniques, thermal evaporation has been the most common one because of its cheap apparatus, widely applicable materials, and convenient operations. A useful approach in the thermal evaporation technique is the use of potassium salts as a catalyst. It was found that the existence of K element can dramatically lower the synthesizing temperature from 1200 to $\sim 2000{ }^{\circ} \mathrm{C}$ to around $600{ }^{\circ} \mathrm{C} .{ }^{11}$ Therefore, this approach has been used to synthesize various tungsten oxide nanostructures, such as nanoribbons and microwalls, except for nanosheets in large scale. ${ }^{10-15}$ In this work, we report a modified simple method for synthesizing highly oriented nanosheets in large scale. This method involves heating tungsten (W) metal with potassium hydrate $(\mathrm{KOH})$ in air via a two-stage heating process. ${ }^{12}$ The obtained nanosheets were characterized by several techniques and the characterization results were discussed. The possible growth mechanism was also proposed.

\section{EXPERIMENTAL}

A tungsten substrate with purity of $99.95 \%$ (Goodfellow, Hungtingdon, UK) and with a size of $5 \mathrm{~mm} \times 5 \mathrm{~mm}$ $\times 0.1 \mathrm{~mm}$ was ultrasonically cleaned consequentially in

\footnotetext{
a) Address all correspondence to this author.

e-mail: hswu@hkusub.hku.hk

DOI: 10.1557/JMR.2009.0026
}

hydrochloric acid, deionized water, ethanol, and deionized water again. It was then immersed into $10 \%$ (wt\%) $\mathrm{KOH}$ solution and heated up by a heating table. When tiny $\mathrm{KOH}$ crystals began to appear on its surface, after the solution boiled, the tungsten substrate was taken out. It was then loaded into a horizontal quartz tube mounted in a conventional horizontal tube furnace. The furnace was then heated from room temperature to 390 ${ }^{\circ} \mathrm{C}$ at a ramping rate of $30{ }^{\circ} \mathrm{C} \mathrm{min}{ }^{-1}$ and maintained at this temperature for $1 \mathrm{~h}$. The temperature was further increased to $610{ }^{\circ} \mathrm{C}$ and kept at this temperature for $2 \mathrm{~h}$. Finally, the furnace was cooled freely down to room temperature by turning off the heating power. During the entire process, the quartz tube was kept to atmosphere. In addition, samples were also prepared under other growth conditions, such as different dipping times of $\mathrm{KOH}$ pretreatment, growth temperature, and gas pressure.

After rinsing softly with deionized water the sample was characterized by scanning electron microscopy (SEM), energy dispersive $\mathrm{x}$-ray (EDX), x-ray diffraction (XRD), transmission electron microscopy (TEM), and Raman scattering spectroscopy. SEM images were taken by using a LEO 1530 Field Emission (Oberkochen, Germany) SEM at $5 \mathrm{kV}$ and the EDX was attached to the SEM. XRD was taken on a Siemens (Huber, Germany) D5000 advance x-ray diffractometer with a $2 \theta$-angle range from $20^{\circ}$ to $80^{\circ}$. High-resolution TEM (HRTEM) images and a selected-area electron diffraction (SAED) pattern were obtained with TECNAI (Eindhoven, The Netherlands) 20 using an accelerating voltage of $200 \mathrm{kV}$. The Raman spectra were measured by a Renishaw (Gloucestershire, UK) RM-1000 microspectrometer with the 514-nm line of an Ar ion laser.

\section{RESULTS AND DISCUSSION}

Figures 1(a)-1(d) are SEM images of a typical sample at different magnifications, which show the morphology 
of the resulted tungsten oxide nanosheets. The nanosheets have thicknesses of $30 \sim 50 \mathrm{~nm}$ [Fig. 1(d)] and widths up to tens of micrometers. A special feature of these nanosheets is that they all have a characteristic acute angle of about $37^{\circ}$ or $51^{\circ}$, respectively. Another phenomenon is that these nanosheets appear on both the top and bottom surfaces of the tungsten substrate, but their quantities on the top surface are a little bit larger than that on the bottom surface. This phenomenon is different from earlier reports in which tungsten oxide appeared only on the top surface of the substrate.

Figure 2 shows the XRD spectra of the grown nanosheets. All diffraction peaks in the spectrum, except for the one at $73.33^{\circ}$, can be well indexed to the peaks of the known primitive structure of tungsten oxide $\mathrm{WO}_{3}$ with lattice constants $a=0.7310 \mathrm{~nm}, b=0.7524 \mathrm{~nm}$, $c=0.7685 \mathrm{~nm}$, and $\beta=90.913^{\circ}$ (JCPDS Card No. 830949). The peak at $73.33^{\circ}$ corresponds to the [211] direction of tungsten substrate (JCPDS Card No. 04-0806). Its appearance is due to the thin nanosheets that cannot completely block the x-ray from reaching the substrate. No peaks associated with other compounds were detected, which implies the high purity of the grown nanosheets. From the strong similarity of the two spectra taken from the top surface [panel (a)] and from the bottom surface [panel (b)], respectively, it is obvious that almost identical nanosheets of tungsten oxide were grown upwards and downwards. The strongest diffraction peaks (020), (040), and (060) in the spectra suggest that [020] is one of the major growth directions of the tungsten oxide nanosheets. The reason for the absence of (010) diffraction peak in the spectra is that the $2 \theta$ scan angle in our measurement starts from $20^{\circ}$, which is larger than the angle for the (010) peak to appear, $11.76^{\circ}$. Furthermore, the EDX spectrum (not displayed here) showed that the grown sample contains only elements $\mathrm{K}, \mathrm{W}$, and $\mathrm{O}$ with a molar ratio of 0.3:1:2.6. While the $\mathrm{K}$ element could be from the residue catalyst, the ratio of $\mathrm{W}$ to $\mathrm{O}$, considering the accuracy of EDX it is close to the suggested structure $\mathrm{WO}_{3}$.

The HRTEM images with geometrical configuration of the grown nanosheets are shown in Fig. 3. The inset of Fig. 3(a) shows one of the two characteristic angles of a typical nanosheet, and it is about $51^{\circ}$. In Fig. 3(b), the plane spacings along (002) and (020) directions are 0.383 and $0.375 \mathrm{~nm}$, respectively, which match the parameters of the structure of $\mathrm{WO}_{3}$. The TEM result together with the SAED pattern as an inset in Fig. 3(b) suggest that [020] and [002] are two major growth directions for the nanosheet, which matches very well with the XRD result.

The characteristic angles of $51^{\circ}$ and $37^{\circ}$ (not shown here) suggest that there exists a third major growth direction that forms either the characteristic angle of $51^{\circ}$ with the [002] surface or the angle of $37^{\circ}$ with the [020] surface. To derive the third growth direction, a TEM image was chosen [Fig. 4(a)] to clearly show the nanosheet boundary along the third direction. In the

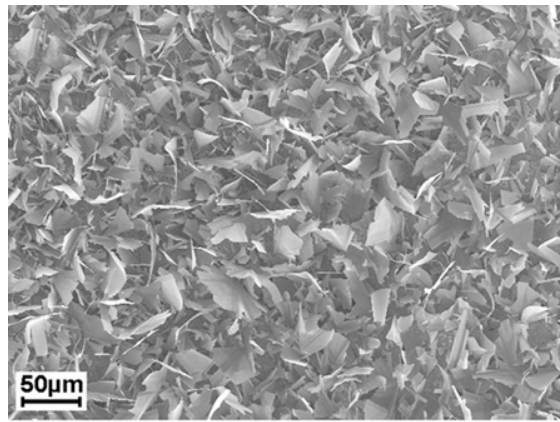

(a)

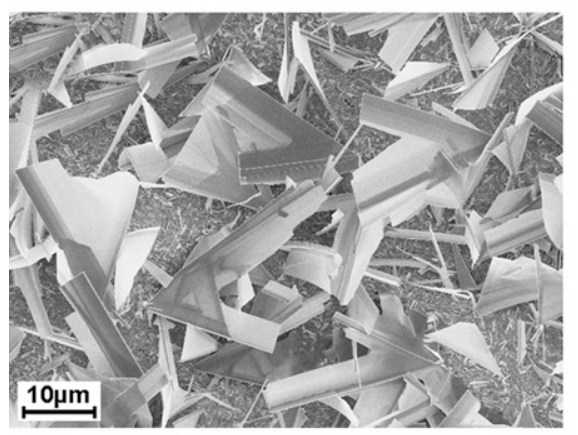

(c)

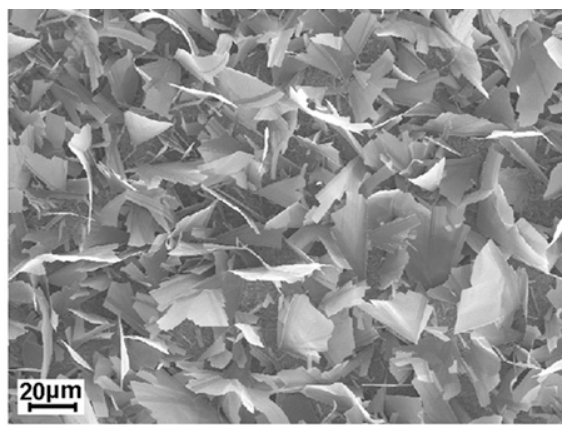

(b)

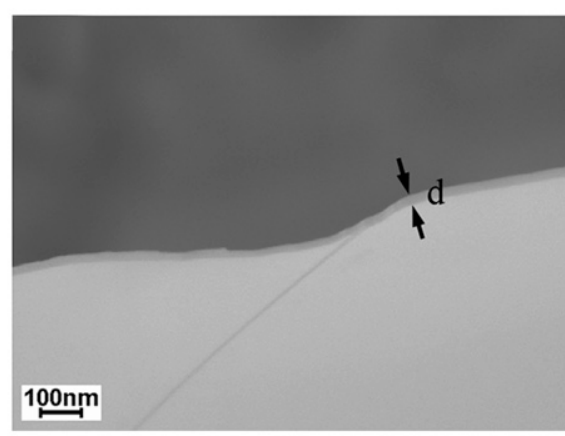

(d)

FIG. 1. SEM images at different magnifications [panels (a)-(c)] with a thickness of $30 \mathrm{~nm}$ [panel (d)]. 
figure, two straight lines were drawn along the [020] and [002] directions, respectively, with the straight line lengths intercepted by the boundary being marked as a and $b$. It was found that the ratio $a / b$ is 0.762 . Taking into account the plane spacings along [020] and [002], the third growth direction is found to be [043]. Unfortunately, there is no XRD peak associated with this direction according to the XRD data base. The theoretical length ratio of $a / b$ is 0.766 , very close to the measured value. The exact values of the angles between [043] and the other two major growth directions are $37.1^{\circ}$ and $52.0^{\circ}$, respectively, close to the observed angles. The detailed configuration among the three growth directions are shown in Fig. 4(b) with the dots representing lattice points.

Raman scattering spectroscopy was also used to investigate the grown tungsten oxide. The Raman spectra shown in Fig. 5 were taken from both the top surface

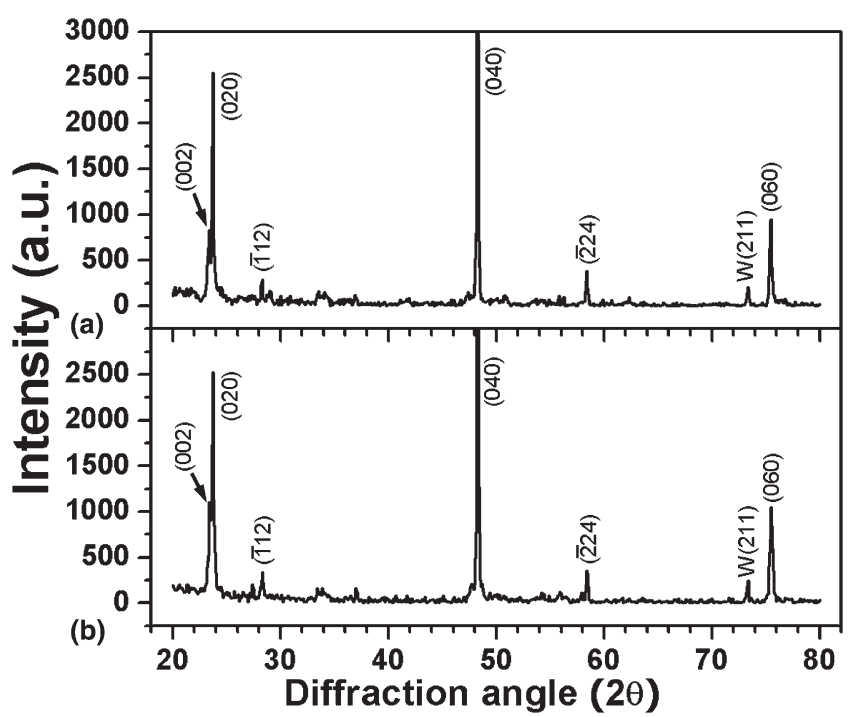

FIG. 2. XRD spectra of the sample: (a) from the top surface; (b) from the bottom surface. [panel (a)] and bottom surface [panel (b)] of the sample with a conventional photon counting system. The two spectra from the two surfaces of the sample are almost the same except for the intensity. The two strongest peaks at 810 and $720 \mathrm{~cm}^{-1}$ are assigned to the stretching vibration of tungsten atom with neighboring oxygen atoms $[\mathrm{v}(\mathrm{O}-\mathrm{W}-\mathrm{O})]$. The peak at around $276 \mathrm{~cm}^{-1}$ accounts for the bending vibration $[\delta(\mathrm{O}-\mathrm{W}-\mathrm{O})]$. The peak at around $965 \mathrm{~cm}^{-1}$ is attributed to the terminal $-\mathrm{W}=\mathrm{O}$ bonds, which are referred to in the literature as the presence of a nanocrystalline structure. ${ }^{16}$ Therefore, the Raman spectrum suggest that the grown tungsten oxide nanosheets are highly nanocrystalline, composed of the octahedral $\mathrm{O}-\mathrm{W}-\mathrm{O}$ units with terminal $-\mathrm{W}=\mathrm{O}$ bonds on the boundaries.

To investigate the role of the dipping of the tungsten plate in the boiling $\mathrm{KOH}$ solution during the preparation, a series of preparation conditions were tested. It was found that the tungsten oxide nanosheets exist only under the condition that the tungsten plate was taken out of the $\mathrm{KOH}$ solution when tiny $\mathrm{KOH}$ crystals just began to appear on its surfaces. There were no tungsten oxide nanosheets existing if the tungsten plate was taken out too early or too late. The possible reason is the following. When the tungsten plate was taken out too early there were no $\mathrm{KOH}$ crystals appearing at all, which prevented the formation of catalyst composed of $\mathrm{K}, \mathrm{W}$, and O. However, if it was taken out too late there were too many $\mathrm{KOH}$ crystals appearing. This was also harmful to the formation of a catalyst because the amount of K may exceed the suitable portion among $\mathrm{K}, \mathrm{W}$, and $\mathrm{O}$ elements required to form the catalyst.

In addition, the effects of different growth conditions on the growth of the nanosheets were also investigated. It was found that the nanosheets can only be obtained when the substrate temperature during growth is in the range of $610 \sim 650{ }^{\circ} \mathrm{C}$. When growing under different gas pressures (from 1 Torr to atmosphere), nanosheets with

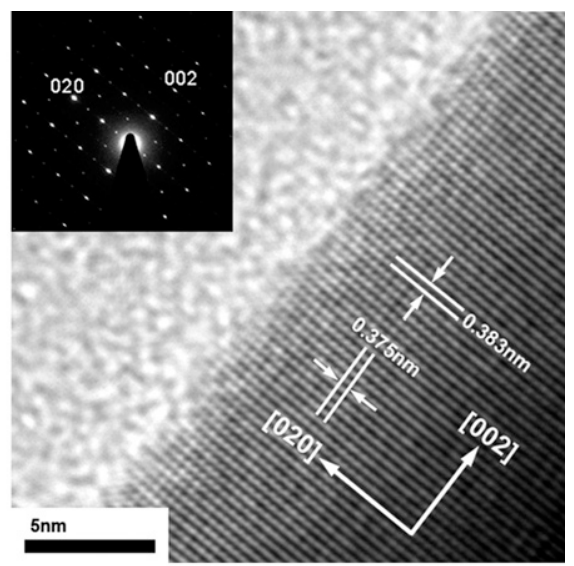

(b) (a)

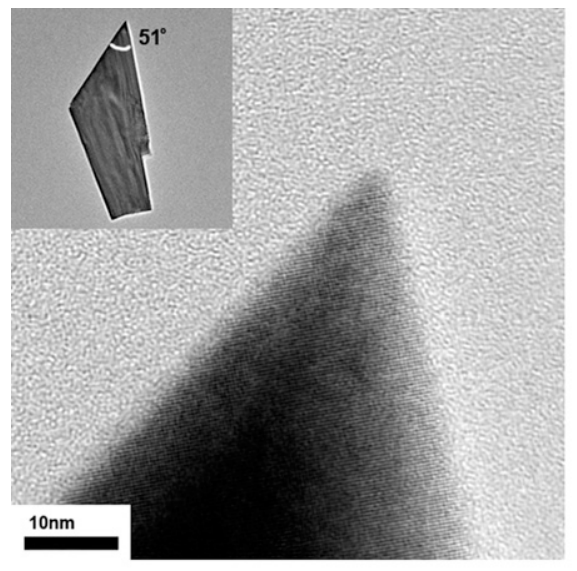




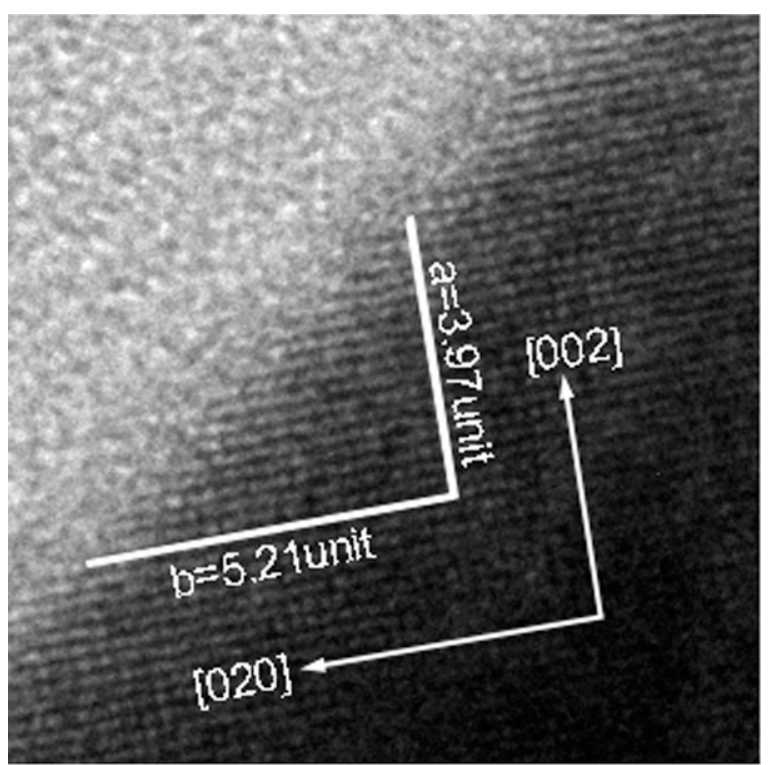

(a)

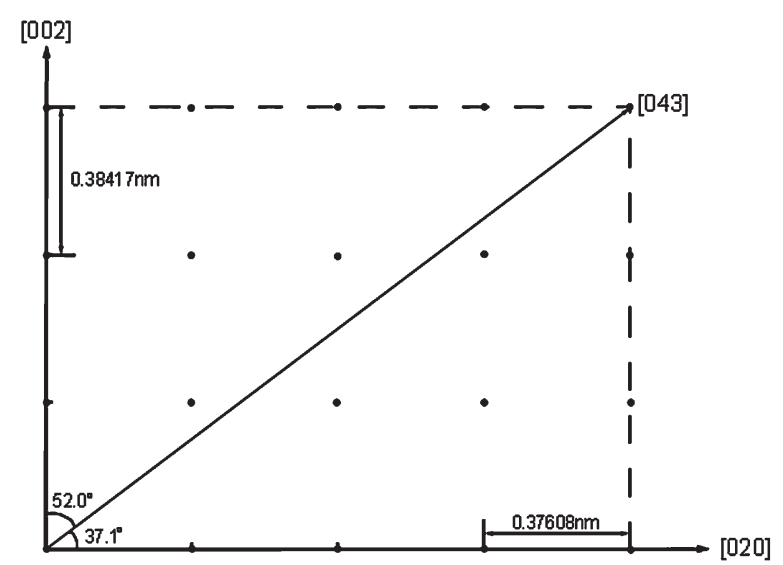

(b)

FIG. 4. Three major growth directions of the nanosheet.

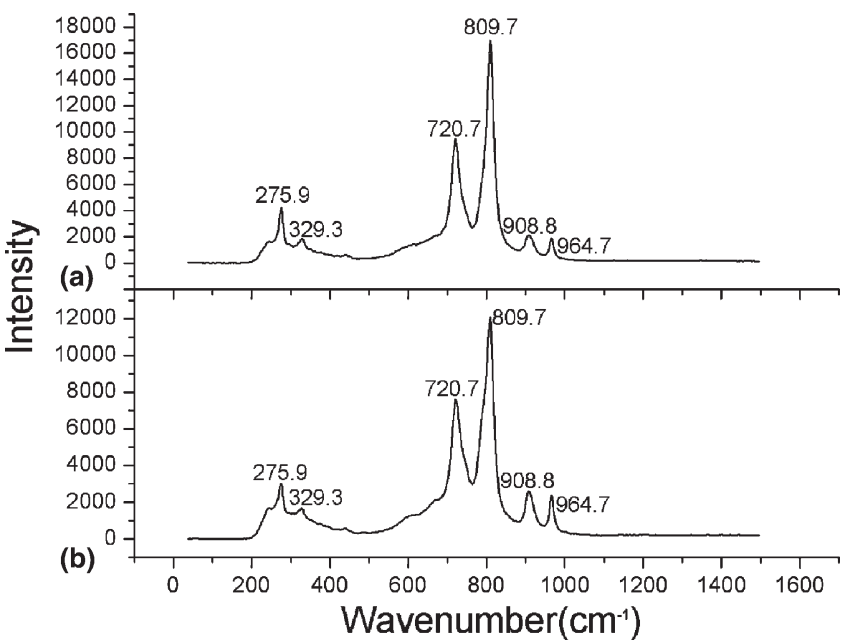

FIG. 5. Raman spectra of the sample from (a) the top surface; and (b) the bottom surface. similar morphology were obtained. This implies that the synthesis is insensitive to gas pressure in quartz tube during the growth.

Considering the characterization results and the fact that the nanosheets were obtained on both surfaces of the tungsten substrate, the vapor-liquid-solid (VLS) mechanism looks appropriate for the growth of the tungsten oxide nanosheets. When the tungsten plates covered with tiny $\mathrm{KOH}$ crystals from the pretreatment were kept for a certain period at $390{ }^{\circ} \mathrm{C}$, the $\mathrm{KOH}$ crystals with a melting temperature of $380{ }^{\circ} \mathrm{C}$ were in a liquid phase and thus they distributed more uniformly on the surface of tungsten substrate. ${ }^{12}$ When the temperature increased to $610{ }^{\circ} \mathrm{C}$, a compound containing $\mathrm{K}, \mathrm{W}, \mathrm{O}$ was formed and was in liquid phase, according to the phase diagram of the K-W-O system. ${ }^{11}$ Sublimated tungsten oxides from some parts of the sample were absorbed to this compound and when supersaturated they began to crystallize at the boundary between the tungsten substrate and the $\mathrm{K}-\mathrm{W}-\mathrm{O}$ compound. This process continued to form nanosheets. ${ }^{17,18}$ The growth temperature of $610{ }^{\circ} \mathrm{C}$ was determined by the composition of the compound and the phase diagram of $\mathrm{K}-\mathrm{W}-\mathrm{O}$ system.

\section{CONCLUSION}

In summary, a large quantity of tungsten oxide nanosheets was synthesized by thermally oxidizing tungsten plates with potassium hydrate as the catalyst and tungsten plates as the substrate. The obtained tungsten oxide nanosheets have thicknesses of 30-50 nm and widths up to tens of micrometers. There exist two characteristic acute angles of about $37^{\circ}$ or $51^{\circ}$ on the sheet plane, which were found to be formed by the two major growth planes (020) or (002) with the third growth plane [043]. Proper dipping time of the tungsten plate in $\mathrm{KOH}$ solution during the preparation process is found to be important for the nanosheet growth. The probable growth mechanism was also discussed.

\section{ACKNOWLEDGMENT}

This work is financially supported by the Research Grant Council of the Hong Kong Special Administrative Region, China under Grant No. HKU 7047/05P.

\section{REFERENCES}

1. A. Aird and E.K.H. Salje: Sheet superconductivity in twin walls: Experimental evidence of $\mathrm{WO}_{3-x}$. J. Phvs. Condens. Matter 10. L377 (1998).

2. O. Bohnke, M. Rezarzi, B. Vuillemin, C. Bohnke, P.A. Gillet, and C. Rousselot: In situ optical and electrochemical characterization of electrochromic phenomena into tungsten trioxide thin-films. Sol. Energy Mater. Sol. Cells 25, 361 (1992).

3. J.L. Solis, S. Saukko, L. Kish, C.G. Granqvist, and V. Lantto: Semiconductor gas sensors based on nanostructured tungsten oxide. Thin Solid Films 391, 255 (2001). 
4. K. Sayama, K. Mukasa, R. Abe, Y. Abe, and H. Arakawa: Stoichiometric water splitting into $\mathrm{H}_{2}$ and $\mathrm{O}_{2}$ using a mixture of two different photocatalysts and an $\mathrm{IO}_{3}$-/I- shuttle redox mediator under visible light irradiation. Chem. Commun. (Camb.) 2416 (2001).

5. K. Lee, W.S. Seo, and J.T. Park: Synthesis and optical properties of colloidal tungsten oxide nanorods. J. Am. Chem. Soc. 125 , 3408 (2003).

6. Z.W. Liu, Y. Bando, and C.C. Tang: Synthesis of tungsten oxide nanowires. Chem. Phys. Lett. 372, 179 (2003).

7. C.C. Liao, F.R. Chen, and J.J. Kai: $\mathrm{WO}_{3-x}$ nanowires based electrochromic devices. Sol. Energy Mater. Sol. Cells 90, 1147 (2006).

8. A.C. Dillon, A.H. Mahan, R. Deshpande, J.L. Alleman, J.L. Blackburn, P.A. Parillia, M.J. Heben, C. Engtrakul, K.E.H. Gilbert, K.M. Jones, R. To, S-H. Lee, and J.H. Lehman: Hot-wire chemical vapor synthesis for a variety of nano-materials with novel applications. Thin Solid Films 501, 216 (2006).

9. Y.B. Li, Y. Bando, and D. Golberg: Quasi-aligned single-crystalline W18O49 nanotubes and nanowires. Adv. Mater. 15, 1294 (2003).

10. Y.M. Zhao, Y.H. Li, I. Ahmad, D.G. McCartney, Y.Q. Zhu, and W.B. Hu: Two-dimensional tungsten oxide nanowire networks. Appl. Phvs. Lett. 89, 133116 (2006).
11. H. Qi, C.Y. Wang, and J. Liu: A simple method for the synthesis of highly oriented potassium-doped tungsten oxide nanowires. Adv. Mater. 15, 411 (2003).

12. R. Hu, H.S. Wu, and K.Q. Hong: Growth of uniform tungsten oxide nanowires with small diameter via a two-step heating process. J. Crvst. Growth 306, 395 (2007).

13. B. Zhang, J.D. Liu, S.K. Guan, Y.Z. Wan, Y.Z. Zhang, and R.F. Chen: Synthesis of single-crystalline potassium-doped tungsten oxide nanosheets as high-sensitive gas sensors. J. Alloys Compd. 439, 55 (2007).

14. K.Q. Hong, W.C. Yiu, H.S. Wu, J. Gao, and M.H. Xie: A simple method for growing high quantity tungsten-oxide nanoribbons under moist conditions. Nanotechnology 16, 1608 (2005).

15. K.Q. Hong, M.H. Xie, R. Hu, and H.S. Wu: Synthesis of potassium tungstate micro-walls by thermal evaporation. J. Crust. Growth 295, 75 (2006).

16. A. Baserga, V. Russo, F. Di Fonzo, A. Bailini, D. Cattaneo, C.S. Casari, A. Li Bassi, and C.E. Bottani: Nanostructured tungsten oxide with controlled properties: Synthesis and Raman characterization. Thin Solid Films 515, 6465 (2007).

17. R.S. Wagner and W.C. Ellis: Vapor-liquid-solid mechanism of single crystal growth. Appl. Phys. Lett. 4, 89 (1964).

18. Y.Y. Wu and P.D. Yang: Direct observation of vapor-liquid-solid nanowire growth. J. Am. Chem. Soc. 123, 3165 (2001). 